\title{
Separation and characterization of antioxidants from Spirulina platensis microalga combining pressurized liquid extraction, TLC and HPLC-DAD
}

\author{
Laura Jaime ${ }^{1}$, José A. Mendiola ${ }^{1}$, Miguel Herrero $^{2}$, Cristina Soler-Rivas $^{1}$, Susana Santoyo ${ }^{1}$, \\ F. Javier Señorans ${ }^{1}$, Alejandro Cifuentes ${ }^{2}$, Elena Ibáñez ${ }^{2 *}$
}

\begin{abstract}
${ }^{1}$ Sección Departamental Ciencias de la Alimentación, Universidad Autónoma de Madrid, Ciudad Universitaria de Cantoblanco, 28049 Madrid, Spain.

${ }^{2}$ Instituto de Fermentaciones Industriales, CSIC, Juan de la Cierva 3, 28006 Madrid, Spain.
\end{abstract}

Running title: PLE-TLC-HPLC-DAD of antioxidants from microalgae

*Corresponding author: Elena Ibáñez

Phone: 34 915622900; Fax: 34 915644853; E-mail: elena@,ifi.csic.es

\begin{abstract}
Abbreviations:
DAD: Diode array detector

DPPH: 2,2-Diphenyl-1-picrylhydrazyl hydrate

PLE: Pressurized liquid extraction

RP-HPLC: Reversed-phase high performance liquid chromatography

TLC: Thin Layer Chromatography
\end{abstract}

Keywords: Spirulina platensis; pressurized liquid extraction, antioxidant activity, TLC, HPLC-DAD 


\begin{abstract}
A new procedure has been developed to separate and characterize antioxidant compounds from Spirulina platensis microalga based on the combination of pressurized liquid extraction (PLE) and different chromatographic procedures, such as Thin-Layer Chromatography (TLC), at preparative scale, and High Performance Liquid Chromatography (HPLC) with a Diode Array Detector (DAD). Different solvents were tested for PLE extraction of antioxidants from Spirulina platensis microalga. An optimized pressurized liquid extraction process using ethanol (generally recognized as safe, GRAS) as extraction solvent has been obtained that provides natural extracts with high yields and good antioxidant properties. TLC analysis of this ethanolic extract obtained at $115^{\circ} \mathrm{C}$ for 15 min was carried out and the silica layer was stained with a $\mathrm{DPPH}^{\bullet}$ (diphenyl-pycril-hydrazyl) radical solution to determine the antioxidant activity of the different chromatographic bands. Next, these coloured bands were collected for their subsequent analysis by HPLC-DAD revealing that the compounds with the most important antioxidant activity present in Spirulina extracts were carotenoids, as well as phenolic compounds and degradation products of chlorophylls.
\end{abstract}




\section{Introduction}

Spirulina is one of the several alga genera that have attracted special attention due to its importance as human foodstuff and its in-vitro and/or in-vivo functional properties. Among this genus, Spirulina platensis has been extensively grown to obtain a protein-rich material of alimentary use, in populations of Africa and Mexico, or industrial use (blue pigments) [1]. In addition, Spirulina platensis is a potential source of high value compounds with functional properties as e.g., phycocyanins, carotenoids, phenolic acids and $\omega-3$ and $\omega-6$ polyunsaturated fatty acids [2-5].

The growing interest in functional foods continues to raise the demand of new food ingredients obtained by extraction of natural products. In that way, several studies showed that Spirulina platensis or its extracts could possess physiological profits as antioxidant, antimicrobial, anti-inflammatory, antiviral or antitumoral properties [5-9]. Moreover, the use of synthetic antioxidants, such as BHT or BHA, has been called into question due to their suspected activity as promoters of carcinogenesis. Thus, there is a great interest in finding new and safe antioxidants from natural sources [10-11]. Opposite to plant extracts where antioxidant activity has been mainly associated to phenolic compounds [12], carotenoids, phenolic acids, degradation products of chlorophyll and phycocyanins have been isolated from Spirulina platensis extracts being these substances the possible active principles $[4,5,13]$.

This interest in new functional foods (or nutraceuticals) from natural sources, has generated the need of developing new analytical procedures able to both provide adequate extracts and 
characterize them from a biological (e.g., antioxidant activity) and chemical point of view in a fast and easy way.

At present, new extraction methods, as supercritical fluid extraction (SFE) or pressurized liquid extraction (PLE), have been developed to overcome the well-known drawbacks of traditional methods (as e.g., liquid-liquid extraction) such as long extraction times, laboriousness, low selectivity and/or low extraction yields. Moreover, these traditional techniques employ large amounts of toxic solvents. On the other hand, whereas supercritical fluid extraction (SFE) is already frequently used as a clean technique to obtain functional compounds from natural sources, pressurized liquid extraction (PLE) still remains under study, being one of the most promising processes. These extraction techniques provide higher selectivity, shorter extraction times and frequently do not require large amounts of toxic organic solvents [14] compared to traditional extraction processes. Extracts obtained by PLE are a mixture of several compounds; therefore, a separation-identification step has usually to be developed afterward in order to adequately characterize them [15]. Among the different analytical procedures employed to analyze these fractions, micellar electrokinetic chromatography (MEKC) and reverse-phase high-performance liquid chromatography (RPHPLC) both coupled with diode array detection (DAD) have been mainly employed [16, 17]. This can be explained through their compatibility with the labile nature of some extracted compounds and because these techniques provide interesting advantages such as high sensitivity and reproducibility [16] for RP-HPLC and high efficiency and short analysis times for MEKC.

Independently of their advantages and drawbacks, it is evident that HPLC or MEKC do not provide straightforwardly the required information about the biological activity of the extracts 
obtained, as for instance their antioxidant activity. In this sense, thin layer chromatography (TLC) is considered a versatile and efficient technique for the separation and identification of pigments depending on their adsorption properties on chromatographic support (specially the $\mathrm{R}_{\mathrm{f}}$ value). Furthermore, developed TLC plates can be stained with radical solutions (as DPPH or $\mathrm{ABTS}^{\bullet+}$ ) obtaining in that way the required information about antioxidant activity of the chromatographic bands $[18,19]$. Such characteristics, together with its easy use, make this technique still widely applied [20].

The aim of this work was to develop an easy and fast method to investigate the compounds responsible of the antioxidant activity of Spirulina platensis extracts. To do this, a complete analytical procedure is applied based on the next steps: i) pressurized liquid extraction is used to obtain fractions from Spirulina microalga; ii) TLC is used to separate and determine the antioxidant activity of the different fractions; iii) the bands with higher antioxidant activity are collected from preparative TLC plates; and iv) RP-HPLC with diode array detection is employed to provide some initial information about the purity and nature of such collected bands. To our knowledge, this is the first time that such a complete procedure is applied to obtain and study fractions from natural origin.

\section{Materials and methods}

\subsection{Samples}

Microalgae samples (Spirulina platensis, from Algamar, Pontevedra, Spain) consisted of airdried microalgae with $6 \%$ moisture mass, stored under dry and dark conditions. 


\subsection{Chemicals}

TLC plates (silica gel $60 \mathrm{~F}_{254}$ on an aluminum backing) were purchased from Merck (Darmstadt, Germany). 2,2-Diphenyl-1-picryhydrazyl hydrate (DPPH, 95\% purity) was obtained from Sigma-Aldrich (Madrid, Spain). Methanol, ethanol, petroleum ether (bp 40-60 ${ }^{\circ} \mathrm{C}$ ) and acetone were purchased from Panreac Quimica (Barcelona, Spain) and hexane of HPLC grade was from Lab Scan (Dublin, Ireland). $\beta$-carotene and lutein standards as well as ammonium acetate were provided by Fluka (Buchs, Switzerland).

Carotenoids standards isolated from phytoplankton used in LC-DAD ( $\beta$-carotene, zeaxanthin, myxoxantophyll, $\beta$-cryptoxanthin and chlorophyll a) from DHI Water \& Environment (Hørsholm, Denmark).

\subsection{Pressurized Liquid Extraction (PLE)}

Extractions of Spirulina platensis were performed using an Accelerated Solvent Extractor (ASE 200, Dionex, Sunnyvale, CA, USA), equipped with a solvent controller. Three different solvents (i.e. hexane, petroleum ether and ethanol) were used to achieve extracts with different composition from a natural matrix. Moreover, extractions were performed at three different extraction temperatures $\left(60^{\circ} \mathrm{C}, 115^{\circ} \mathrm{C}\right.$ and $\left.170^{\circ} \mathrm{C}\right)$ and extraction times $(3,9$ and 15 minutes). Previously, an extraction cell heat-up was carried out for a given time, which changed according to extraction temperature. All extractions were performed in $11 \mathrm{ml}$ extraction cells, containing $2.5 \mathrm{~g}$ of sample.

Extraction method was performed according to the procedure described by Herrero et al. [15]. The extracts were covered with aluminum foil and stored at $-18^{\circ} \mathrm{C}$ until dried. Solvents were removed under a stream of nitrogen gas. Afterwards, dry extracts were dissolved using the 
same solvent as used during extraction at $30 \mathrm{mg} / \mathrm{ml}$ concentration. In the same way, the solutions were stored at $-18^{\circ} \mathrm{C}$ and protected from light with aluminum foil.

\subsection{TLC}

Analytical-TLC was carried out in TLC plates $(10 \mathrm{~cm} \times 20 \mathrm{~cm})$ cut from the commercially available sheets. $12 \mu \mathrm{l}$ of $30 \mathrm{mg} / \mathrm{ml}$ extract solution were applied to $1 \mathrm{~cm}$ of the base of the silica gel layer and allowed to dry for a few minutes. Afterwards, the plate was eluted in a closed chamber with petroleum ether/acetone $(75 / 25)$ as mobile phase (eluent 1$)$. The staining of the silica layers with $\mathrm{DPPH}^{\bullet}$ radical was based on the procedure of Soler-Rivas et al. [19] with some modifications. The developed plate was placed upside down for $10 \mathrm{~s}$ in a $0.5 \mathrm{mM}$ $\mathrm{DPPH}^{\bullet}$ solution in methanol. After this time, the excess of solution was removed with a tissue paper and the layer was dried with a hair-dryer under cold air.

Preparative-TLC analysis was performed as follows: $300 \mu 1$ of Spirulina platensis extract was carefully placed on the baseline of $20 \times 20 \mathrm{~cm}$ TLC layer. Two different mobile phases were use in order to obtain a better separation of the bands according to their polarity: the first one corresponded to the same mobile phase as in the analytical-TLC (eluent 1) while the other consists of a mixture of toluene/ethyl acetate/formic acid (50/40/10) (eluent 2). In each experiment, two plates were used in parallel. One of the plates was stained with $0.5 \mathrm{mM}$ $\mathrm{DPPH}^{\bullet}$ solution as described above, and those bands that showed antioxidant activity were scraped off from the second plate, eluted from the silica with either hexane (in the case of non-polar carotenoids) or acetone or ethanol (if the polarity was medium) and filtered to remove the silica. 


\subsection{HPLC-DAD analysis}

The scraped bands were analyzed by HPLC using a Hewlett-Packard HP 1090 Liquid Chromatograph equipped with a DAD. HPLC method has been published elsewhere [17]. The separation was carried out in a Novapack $\mathrm{C}_{18}$ column $150 \times 3.9 \mathrm{~mm}, 4 \mu \mathrm{m}$ particle size from Waters. The mobile phase was a mixture of solvent A (Methanol:Ammonium acetate $0.1 \mathrm{~N}$; 7:3 ) and solvent B (Methanol) at $0.9 \mathrm{~mL} / \mathrm{min}$ according to a step gradient, lasting $35 \mathrm{~min}$, which start from $25 \% \mathrm{~B}$, changing at $50 \%$ in one minute, rising up to $100 \% \mathrm{~B}$ at minute 10 , then the mobile phase composition was kept constant until the end of the analysis, total acquisition time $35 \mathrm{~min}$. Injection volume was $20 \mu \mathrm{l}$. The identification of the peaks was performed, when possible, using standards. When no standards were available, tentative identification was done based on UV-vis spectra characteristics and comparing with data appearing in the literature.

\subsection{Antioxidant activity determination (in vitro assay)}

Antioxidant activity was measured in all the extracts using a method based on a procedure described by Brand-Williams et al. [21] and modified by Herrero et al. [15]. Briefly, a solution was prepared dissolving $23.5 \mathrm{mg}$ of DPPH in $100 \mathrm{~mL}$ methanol and stored at $4{ }^{\circ} \mathrm{C}$. This stock solution was diluted 1:10 on methanol. Different concentrations of the extract solutions were prepared. Then $0.1 \mathrm{~mL}$ of these solutions were added to $1.9 \mathrm{~mL}$ diluted $\mathrm{DPPH}^{\bullet}$ solution to complete the final reaction medium $(2 \mathrm{~mL})$. The reaction was completed after $4 \mathrm{~h}$ at room temperature, and the absorbance was measured at $516 \mathrm{~nm}$ in a UV-Vis 6305 spectrophotometer from Jenway (Essex, England). Methanol was used to adjust the zero. The absorbance value was obtained by subtracting the blank absorbance measurement to the value given by the extract solution. A calibration curve was obtained that correlates the concentration of $\mathrm{DPPH}^{\bullet}$ with the Absorbance measured at $516 \mathrm{~nm}$. For each extract five 
different concentration solutions were prepared in order to obtain the remaining $\mathrm{DPPH}^{\bullet}$ concentration upon completion of the reaction. The use of these values allowed the estimation of the extract concentration necessary to achieve a $50 \%$ reduction of the initial $\mathrm{DPPH}^{\bullet}$ concentration, that is, $\mathrm{EC}_{50}$ (Efficient concentration, also called oxidation index). Measurements have been done by duplicate.

\section{Results and discussion}

\subsection{PLE extracts from Spirulina platensis}

A screening of different PLE conditions (solvent, extraction time and extraction temperature) was carried out in order to obtain different composition extracts from Spirulina platensis (see Table 1). The choice of the solvent is based on their different polarity (dielectric constants equal to 1.9 for hexane, 4.3 for petroleum ether and 24.3 for ethanol) and therefore, on their different ability to isolate various groups of compounds that can be responsible of the antioxidant activity of the extracts. Several extraction temperatures $\left(60,115\right.$ and $170^{\circ} \mathrm{C}$; considering always that enough pressure is held to keep the solvents in a liquid state) and times $\left(3,9\right.$ and $15 \mathrm{~min}$ at medium temperature, that is, $\left.115^{\circ} \mathrm{C}\right)$ were tested to study the effect of both factors in the extraction yield (that is, extraction efficiency) and in the degradation of the extracted compounds. Conditions tested were selected also based on previous results obtained in our laboratory [15]. As can be seen, the extraction yield increases by increasing the polarity of the solvent being maximum when working with ethanol (reaching up to $20 \%$, given as dry weight); also, the higher the extraction time and temperature, the higher the extraction yield although the effect of the extraction time is almost negligible (at the evaluated conditions), as we have reported in a previous work where exhaustive optimization of PLE conditions were presented and discussed [22]. 


\subsection{Analysis by TLC}

The analytical-TLC of microalgae extracts shows different bands as can be seen in Figure $1 \mathrm{a}$. (description of the bands as in Table 1). The main pigments in Spirulina platensis microalga have been described $[23,24]$ as chlorophyll a and different carotenoids such as $\beta$-carotene, $\beta$ cryptoxanthin, zeaxanthin, echinenone, oscillaxanthin and myxoxanthophyll. Thus, the orange, yellow and green bands in analytical-TLC of Figure 1 probably would correspond to some of these pigments. Analytical-TLC showed a strong orange band at $\mathrm{R}_{\mathrm{f}}=0.94$, and an intense orange-yellowish band at $R_{f}=0.15$. A yellow band $\left(R_{f}=0.91\right)$, probably an isomer of $\beta$-carotene, and more pale orange or orange-yellowish bands $\left(\mathrm{R}_{\mathrm{f}}=0.84,0.72\right.$ and 0.27$)$ were also found. Several authors have reported $\mathrm{R}_{\mathrm{f}}$ values of different carotenoid compounds, using similar eluents, that seem to agree with our results $[25,26]$. In these studies, $\beta$ - and $\alpha$ carotene bands were always found at the top of the TLC, lutein/zeaxanthin (that elute with the same $\mathrm{R}_{\mathrm{f}}$ ) showed $\mathrm{R}_{\mathrm{f}}$ values close to 0.2 , whereas cryptoxanthin, asthaxanthin and other xanthophylls presented variable middle $R_{\mathrm{f}}$ values depending on their esterification $[25,26]$. In this way, $\beta$-carotene and lutein standards showed $R_{f}=0.94$ and 0.15 , respectively; thus, as zeaxanthin instead lutein has been described in Spirulina platensis microalgae, $\beta$-carotene and zeaxanthin are probably the pigments found in the PLE extracts. Furthermore, green wide bands were found close to the baseline.

The TLC results also showed differences in the extracts composition regarding to the extraction conditions. Hexane and petroleum ether extracts showed similar composition, a higher amount of carotenoids together with lower chlorophyll content, opposite to ethanol extracts behavior. Hexane and petroleum ether extracts showed two intense orange bands at $\mathrm{Rf}=0.94$ and 0.15 together with other orange-yellowish bands, whereas ethanol extracts showed slight orange bands at $\mathrm{Rf}=0.94,0.27$ and 0.15 and a deeper green band. 
Moreover, dark grey-greenish bands were found in the middle of the analytical-TLC $\left(\mathrm{R}_{\mathrm{f}}=\right.$ 0.54 and 0.43$)$ together with other slight grey-greenish band at lower $R_{f}\left(R_{f}=0.35\right)$. These bands might correspond to pheophytin-like compounds that come from the partial degradation of chlorophyll a. Quach et al. [27] reported that extraction methods with organic solvents that rely on high temperatures and/or prolonged periods of extraction results in some pigment degradation. Consequently, PLE caused a partial degradation of chlorophyll a because the loss of magnesium ion from chlorophyll ring resulting in the formation of the dark colored pheophytin at $\mathrm{R}_{\mathrm{f}}$ values higher than the original chlorophyll a. Ethanol was the solvent that caused the lowest formation of pheophytin, whereas the extraction with hexane and petroleum ether lead to similar chlorophyll degradation.

Regarding to the other extraction conditions, pheophytin and chlorophyll bands enhanced by increasing extraction temperature (bands 1, 3, 5 for petroleum ether, $6,8,10$ for hexane and 11, 13 and 15 for ethanol); moreover similar behavior was shown in relation to extraction time independently of the solvent used (bands 2, 3, 4 for petroleum ether, 7, 8, 9 for hexane and $12,13,14$ for ethanol all of them at $115^{\circ} \mathrm{C}$ and 3,9 and $15 \mathrm{~min}$, respectively).

As can be seen in Figure 1.b, stained silica plates with $\mathrm{DPPH}^{\bullet}$ radicals, gave a purple background with bright yellow bands at the locations of those compounds which showed antioxidant activity. The intensity of the yellow color depended on the amount and nature of radical scavengers in the sample. Thus, orange, orange-yellowish and yellow bands showed a brilliant yellow color after the analytical-TLC was dyed with $0.5 \mathrm{mM} \mathrm{DPPH}^{\bullet}$ (see Figure 1. b). The appearance of these vivid color lines implied that these bands showed radical scavenger capacity, as $\mathrm{DPPH}^{\bullet}$ radical (purple) turned out bright yellow (DPPH) in the 
presence of antioxidant compounds. Moreover, pheophytin-like compounds as a degradation product of chlorophyll a, showed a slight antioxidant capacity. Extraction with hexane or petroleum ether at $115^{\circ} \mathrm{C}$ and 15 minutes yielded the more intense spots while ethanolic extracts showed a very intense spot at the origin, where sample was placed, indicating that the most active compounds in terms of antioxidant activity are relatively polar.

On the other hand, as shown in Table 1, antioxidant activity of hexane, ether and ethanol extracts do not differ strongly one from another and the $\mathrm{EC}_{50}$ values (efficient concentration) ranged from around 70 (for hexane and petroleum ether) to around $80(\mu \mathrm{g} / \mathrm{mL})$ for ethanol. Moreover, ethanol has a great advantage, compared to hexane and petroleum ether, since it is considered a GRAS solvent and therefore can be used as a safe solvent for the food industry. In addition, yields obtained with ethanol are the highest (as discussed above, see Table 1) providing a good efficiency of the extraction process and increasing the viability of the process at industrial scale.

Thus, the microalga extract obtained at $115^{\circ} \mathrm{C}$ and 15 minutes using ethanol as solvent was selected for preparative-TLC in order to further characterize the antioxidant compounds of Spirulina platensis extracts. Furthermore, as mentioned above, in order to identify the more polar compounds (that seem responsible for the antioxidant activity of the ethanolic extracts), 2 different phases with complementary polarities have been used as eluents (eluent 1 and 2).

Preparative-TLC of ethanolic extracts (see Figure 2.a and 3.a) yielded several distinct bands showing high antioxidant activity after staining with $0.5 \mathrm{mM} \mathrm{DPPH}^{\bullet}$ (Figure 2.b and 3.b). These bands, namely 1.1 to 1.5 for eluent 1 (Figure 2) and 2.1 to 2.3 for eluent 2 (Figure 3) were recovered for their subsequent analysis by RP-HPLC-DAD. The bands were scraped off, 
eluted from the silica with either hexane, acetone or ethanol (depending on their polarity) and filtered to remove the silica.

\subsection{Analysis by HPLC-DAD}

Ethanolic extract obtained at optimal conditions $\left(115^{\circ} \mathrm{C}\right.$ and $\left.15 \mathrm{~min}\right)$ was also analyzed by HPLC-DAD to obtain a chromatographic profile of the compounds that are present in the whole extracts (Figure 4). Only few of these compounds have antioxidant capacity and therefore preparative-TLC was used to isolate those bands able to react with the free radical $\mathrm{DPPH}^{\bullet}$, as explained above. The scraped bands of the preparative TLC were analyzed by HPLC-DAD allowing a preliminary characterization of the compounds (Figures 5 and 6) based on their retention time and UV-Vis spectra by comparison with standards, when available, or with data appearing in the literature and previous work done in our laboratory [17]. Figure 7 shows the UV-vis spectra of the compounds that have been detected in the Spirulina extracts.

Figure 5.a, b and c shows the chromatograms corresponding to the bands 1.1, 1.2 and 1.4 isolated using eluent 1 , which, as mentioned, has a lower polarity than eluent 2 . As can be seen, different carotenoids are isolated with good selectivity in the different bands. For instance, Figure 5.a shows, as expected, the separation of $\beta$-carotene that eluted in the TLC with a Rf equal to 0.94 ; in this fraction other carotenoids (not identified) are separated along with $\beta$-carotene that corresponded to those eluting close to this compound in the TLC, even though no complete identification of these peaks is possible, it is likely to assume that these compounds have a molecular structure very similar to that of $\beta$-carotene. Figure 5.b and 5.c show the isolation of zeaxanthin and a polar carotenoid not identified, respectively. The nonidentified polar carotenoid seems to have an structure derived from myxoxanthophyll with UV maxima at 450, 480 and $510 \mathrm{~nm}$ [28]. These carotenoids seem to contribute to the total 
antioxidant activity of the Spirulina extract since they correspond to the bands that react with $\mathrm{DPPH}^{\bullet}$ with higher intensity. The other band (corresponding to 1.3 ) was also analyzed by HPLC but no information could be obtained from them probably due to the low concentration of these bands.

Bands corresponding to the fractions 2.1 through 2.3 (eluent 2) were analyzed by HPLC-DAD and the corresponding chromatograms are shown in Figure 6.a, b and c. It is interesting to notice that the eluent 2 has a higher polarity thus allowing a better separation and fractionation of band 1.5 (that remained close to the origin using eluent 1). As can be seen, chromatograms of the three major bands with antioxidant capacity scraped off after TLC with eluent 2 show the separation and isolation of zeaxanthin (Figure 6.a), non-identified polar carotenoid, probably an structure derived from myxoxanthophyll (Figure 6.b) and phenolic compounds (Figure 6.c) whose chromatogram is shown at $280 \mathrm{~nm}$ that is the maximum wavelength for such family of compounds. This fact confirms the presence of very polar phenolic compounds (whose UV-Vis spectra are consistent with those of $\mathrm{C}_{6}-\mathrm{C}_{1}$ or $\mathrm{C}_{6}$ phenolic skeletons [29]). These compounds appear as a coloured blue band of Rf equal to 0.15. Further studies are conducted to obtain an unequivocal identification of the phenolic compounds found in the ethanolic extracts of Spirulina. This is of great importance considering that little is known about the presence of such compounds in Spirulina.

\section{Concluding remarks}

In this work, it has been demonstrated that the use of the combined protocol PLE-TLCHPLC-DAD allows in a fast and simple way the extraction, purification and preliminary characterization of several antioxidant compounds from Spirulina platensis microalga. Since the complete PLE-TLC-HPLC-DAD procedure once optimized takes less than three hours, it 
is anticipated that this procedure will be applied to study other natural sources of functional ingredients with antioxidant activity.

\section{Acknowledgements}

JAM and MH thank MCYT for FPI grants. Authors thank MCYT (project AGL2002-04621C02-02) for financial support. 


\section{References}

[1] J.C. Dillon, A.P. Phuc, J.P. Dubacq, World Rev. Nutr. Diet. 1995, 77, 32-46.

[2] M. Careri, L. Furlattini, A. Mangia, M. Musci, E. Anklam, A. Theobald, C. Holst, J. Chromatography A. 2001, 912, 61-71.

[3] R.L. Mendes, B.P. Nobre, M.T. Cardoso, A.P. Pereira, A. F. Palabra. Inorg. Chim. Acta. 2003, 356, 328-334.

[4] M.S. Miranda, R.G. Cintra, S.B.M. Barros, F.J. Mancini. Braz. J. Med. Biol. Res. 1998, $31,1075-1079$.

[5] J.E. Piñero-Estrada, P. Bermejo-Bescós, A. M. Villar-del Fresno, Il Farmaco. 2001, 59, 497-500.

[6] J. Subhashini, S.V.K. Mahipal, M.C. Reddy, M.M. Reddy, A. Rachamallu, P. Reddanna, Biochem. Pharmacol. 2004, 68, 453-462.

[7] T. Hirahashi, M. Matsumoto, K. Hazeki, Y. Saeki, M. Ui, T. Seya, Int. Immunopharmacol. 2002, 2, 423-434.

[8] G. Ozdemir, N.U. Karabay, M. C. Dalay, B. Pazarbasi, Phytother. Res. 2004, 18, 754-757.

[9] K. Hayashi, T. Hayashi, N. Morita, Phytother. Res. 1993, 7, 76-80.

[10] M. Namiki, Crit. Rev. Food Sci. Nutr. 1990, 29, 273-300.

[11] J. Pokorny, Trends Food Sci. Technol. 1991, 9, 223-227.

[12] W. Zheng, S. Y. Wang, J. Agric. Food Chem. 2001, 49, 5165-5170.

[13] M.C.Z. Demule,G.Z. Decaire, M.S. Decano, J. Exp. Bot. 1996, 58, 93-96.

[14] J.W. King, Food Sci. Technol. Int. 2000, 14, 186-189.

[15] M. Herrero, E. Ibáñez, J. Señorans, A. Cifuentes. J. Chromatogr. A. 2004, 1047, 195203.

[16] C. Simó, E. Ibañez, F.J. Señorans, C. Barbas, G. Reglero, A. Cifuentes. J. Agric. Food Chem. 2002, 50, 6648-6652. 
[17] J.A. Mendiola, F.R. Marín, S.F. Hernández, B.O. Arredondo, F.J. Señoráns, E. Ibañez, G. Reglero, J. Sep. Sci. 2005, In press.

[18] T. Takao, F. Kitatani, N Watanabe, A. Yagi, K. Sakata. Biosci. Biotechnol. Biochem. 1994, $58,1780-1783$.

[19] C. Soler-Rivas, J.C. Espín, H.J. Wichers. Phytochem. Analysis. 2000, 11, 330-338.

[20] J. Sherma. J Chromatogr. A. 2000, 880, 129-147.

[21] W. Brand-Williams, M.E. Cuvelier, C. Berset, Lebensm.-Wiss. U.-Technol. 1995, 28, $25-$ 30.

[22] M. Herrero, P. J. Martín-Álvarez, F.J. Señoráns, A. Cifuentes, E. Ibáñez, Food Chem. 2005, In press (available online)

[23] C. Van den Hoek, D.G. Mann, H.M. Jahns. Algae: an introduction to phycology, Cambridge University Press 1995, pp. 12-13.

[24] T. Gireesh, A. Jayadeep, K.N. Rajasekharan, V.P. Menon, M. Vairamany, G. Tang, P.P. Nair, P.R. Sudhakaran. Biotechnol. letters. 2001, 23, 447-449.

[25] N.M. Sachindra, N. Bhaskar, N.S. Mahendrakar. Lebensm.-Wiss. U.-Technol. 2005, In press (available online).

[26] T. Hodisan, C. Socaciu, I. Ropau, G. Neamtu. J. Pharmaceut. Biomed. 1997, 16, 521528.

[27] H.T. Quach, R.L. Steeper, G.W. Griffin. J. Chem. Educ. 2004, 81, 385-387.

[28] S. Hertzberg, S. Liaaen-Jensen, Phytochem. 1969, 8, 1259-1280

[29] K. R. Markham, Techniques of flavonoid identification. London, Academic Press, 1982. 
Table 1. PLE conditions (solvent, extraction time and extraction temperature) of Spirulina platensis microalga; extraction yields $(\%)$ and antioxidant activities $(\mu \mathrm{g} / \mathrm{mL})$. Some of the data has been taken from reference [22]. Row number corresponds to their position in the analytical-TLC (Figure 1).

\begin{tabular}{|c|c|c|c|c|c|}
\hline Row & Solvent & $\begin{array}{c}\text { Extraction } \\
\text { temperature } \\
\left({ }^{\circ} \mathrm{C}\right)\end{array}$ & $\begin{array}{l}\text { Extraction } \\
\text { time (min) }\end{array}$ & Yield (\%) & $\begin{array}{c}\text { Antioxidant } \\
\text { Activity } \\
\mathrm{EC}_{50}(\mu \mathrm{g} / \mathrm{ml})\end{array}$ \\
\hline 1 & \multirow{5}{*}{$\begin{array}{c}\text { PETROLEUM } \\
\text { ETHER }\end{array}$} & 60 & 9 & 0.43 & 82.7 \\
\hline 2 & & 115 & 3 & 1.32 & 76.8 \\
\hline 3 & & 115 & 9 & 1.58 & 74.6 \\
\hline 4 & & 115 & 15 & 1.66 & 74.9 \\
\hline 5 & & 170 & 9 & 2.94 & 109.0 \\
\hline 6 & \multirow{5}{*}{ HEXANE } & 60 & 9 & 0.5 & 82.7 \\
\hline 7 & & 115 & 3 & 1.43 & 74.7 \\
\hline 8 & & 115 & 9 & 1.76 & 73.1 \\
\hline 9 & & 115 & 15 & 1.77 & 72.9 \\
\hline 10 & & 170 & 9 & 4.28 & 110.3 \\
\hline 11 & \multirow{5}{*}{ ETHANOL } & 60 & 9 & 7.28 & 98.8 \\
\hline 12 & & 115 & 3 & 12.33 & 84.8 \\
\hline 13 & & 115 & 9 & 11.54 & 86.5 \\
\hline 14 & & 115 & 15 & 11.94 & 89.2 \\
\hline 15 & & 170 & 9 & 19.70 & 100.1 \\
\hline
\end{tabular}




\section{Figure legends}

Figure 1. a) Analytical-TLC of Spirulina platensis PLE extracts; b) Analytical-TLC of Spirulina platensis PLE extracts stained with $0.5 \mathrm{mM} \mathrm{DPPH}^{\bullet}$ solution.

Figure 2. a) Preparative-TLC of ethanol PLE extracts ( $\left.115^{\circ} \mathrm{C}, 15 \mathrm{~min}\right)$; b) Preparative-TLC of ethanol PLE extracts $\left(115^{\circ} \mathrm{C}, 15 \mathrm{~min}\right)$ stained with $0.5 \mathrm{mM} \mathrm{DPPH}^{\bullet}$ solution. Eluent 1: petroleum ether/acetone $(75 / 25)$.

Figure 3. a) Preparative-TLC of ethanol PLE extracts $\left(115^{\circ} \mathrm{C}, 15 \mathrm{~min}\right)$; b) Preparative-TLC of ethanol PLE extracts $\left(115^{\circ} \mathrm{C}, 15 \mathrm{~min}\right)$ stained with $0.5 \mathrm{mM} \mathrm{DPPH}^{\bullet}$ solution. Eluent 2: toluene/ethyl acetate/formic acid (50/40/10).

Figure 4. HPLC-DAD $440 \mathrm{~nm}$ chromatogram corresponding to the ethanol PLE extracts $\left(115^{\circ} \mathrm{C}, 15 \mathrm{~min}\right)$. Chromatographic method: Novapack C18 column $150 \times 3.9 \mathrm{~mm}, 4 \mu \mathrm{m}$ particle size; mobile phase: solvent A (Methanol:Ammonium acetate $0.1 \mathrm{~N} ; 7: 3$ ), solvent B (Methanol), gradient (35 min) starting from $25 \%$ B to $50 \%$ in one minute, rising up to $100 \%$ $\mathrm{B}$ at minute 10. Flow rate $0.9 \mathrm{~mL} / \mathrm{min}$ Identification: unidentified polar carotenoid (1), zeaxanthin (2), unidentified carotenoid (3), myxoxantophill fucoside (4), chlorophylls (5-9), unidentified carotenoids $(10,11)$ and $\beta$-carotene isomers (12).

Figure 5. Chromatogram profile of scraped TLC bands with antioxidant activity (Fig. 2). LC conditions and identification as in Fig. 4 plus peak (8.b), non-identified carotenoid. Chromatograms $a, b$ and $c$ correspond to TLC bands eluted with phase eluent 1; bands 1.1, 1.2 , and 1.5 . 
Figure 6. Chromatogram profile of scraped TLC bands with antioxidant activity (see Fig. 3). LC conditions and identification as in Fig. 4 plus peaks from (13) to (15), phenolic compounds. Chromatograms $a, b$ and $c$ correspond to bands eluted with phase eluent 2; bands 2.1, 2.2 and 2.3. All chromatograms are shown at $440 \mathrm{~nm}$ except $c$ that is given at $280 \mathrm{~nm}$.

Figure 7. UV-vis spectra of the compounds detected in the ethanol PLE extract. Numbers correspond to those in Figure 4. 


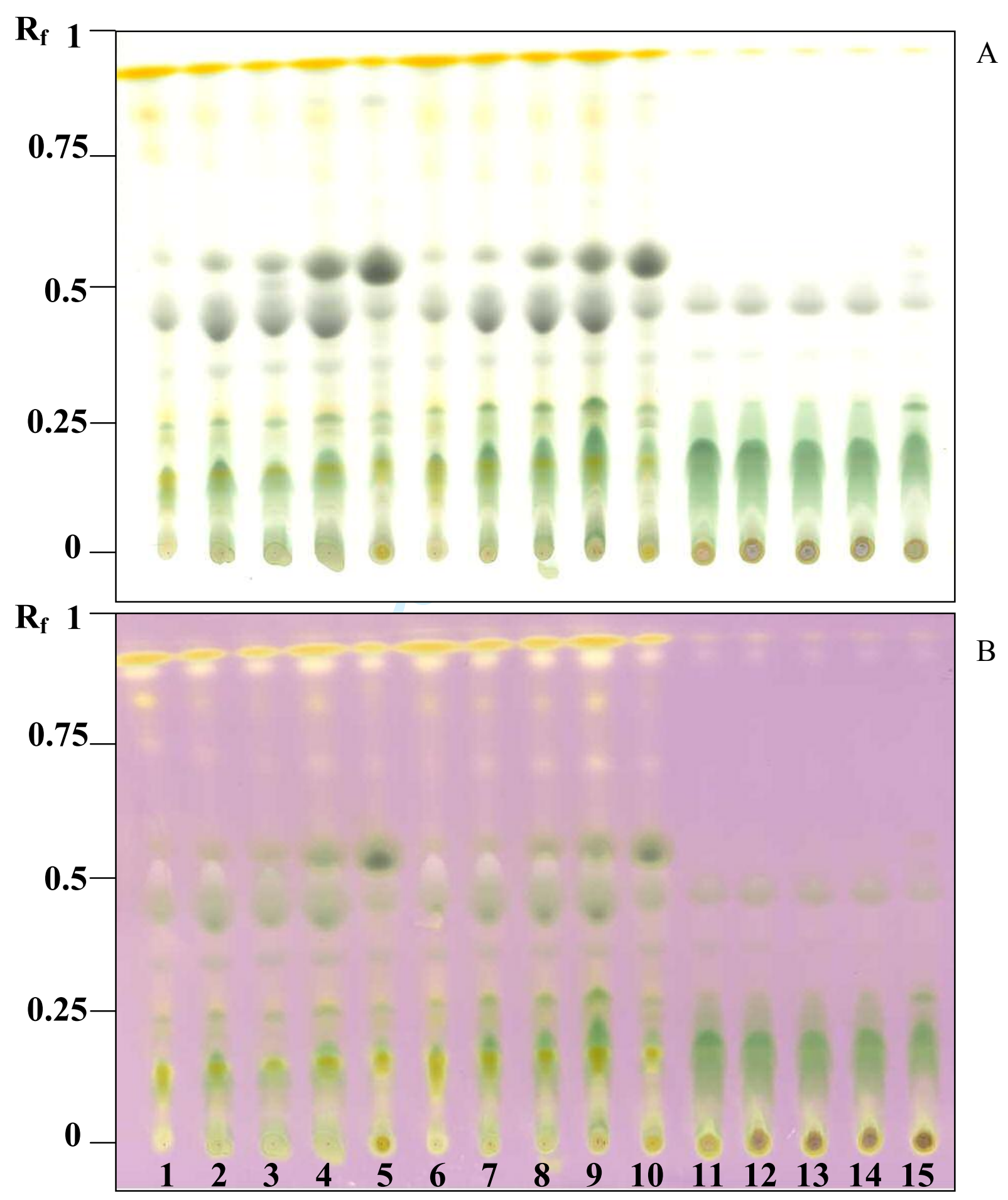

Figure 1. 
A

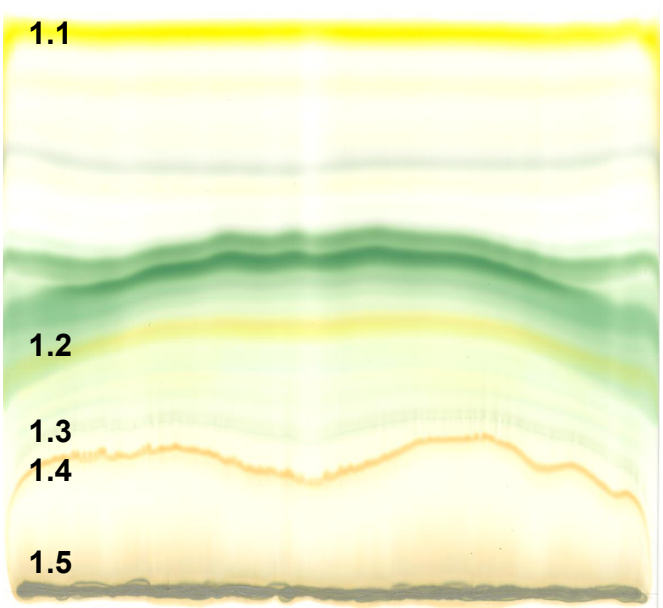

B

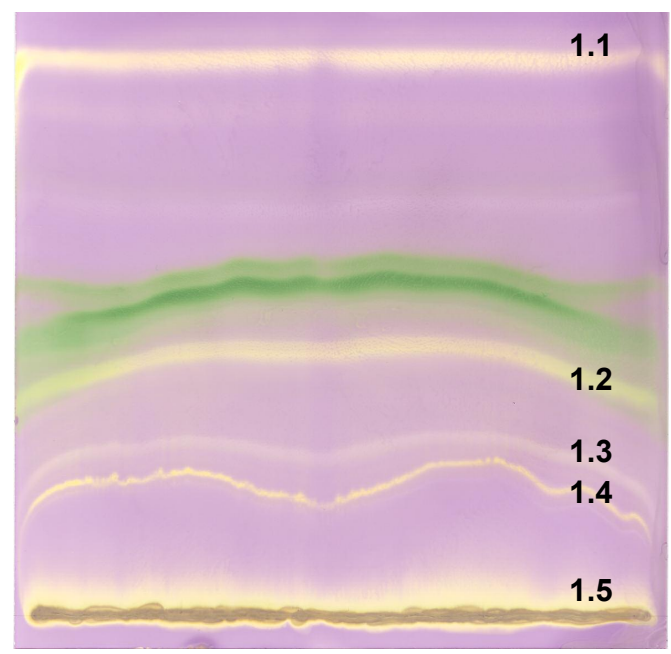

Figure 2.

Wiley-VCH 
A

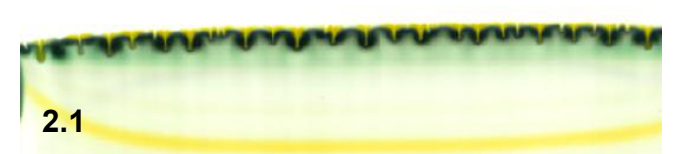

2.2

2.3

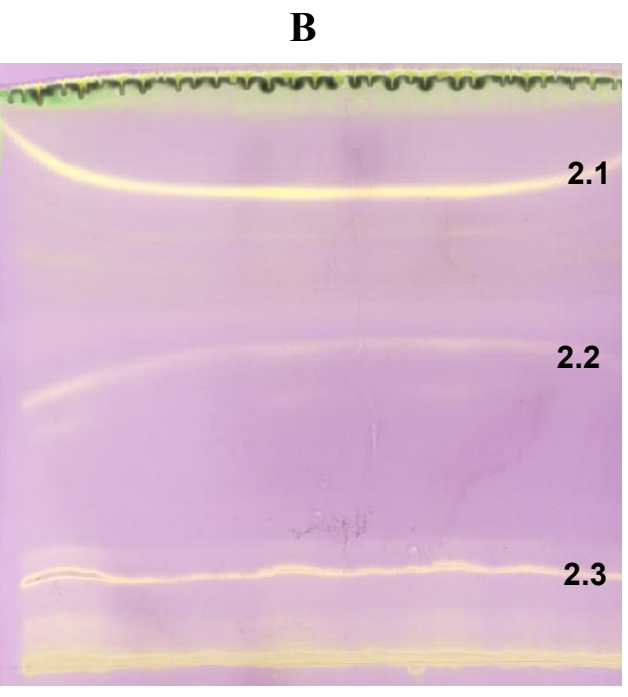

Figure 3. 


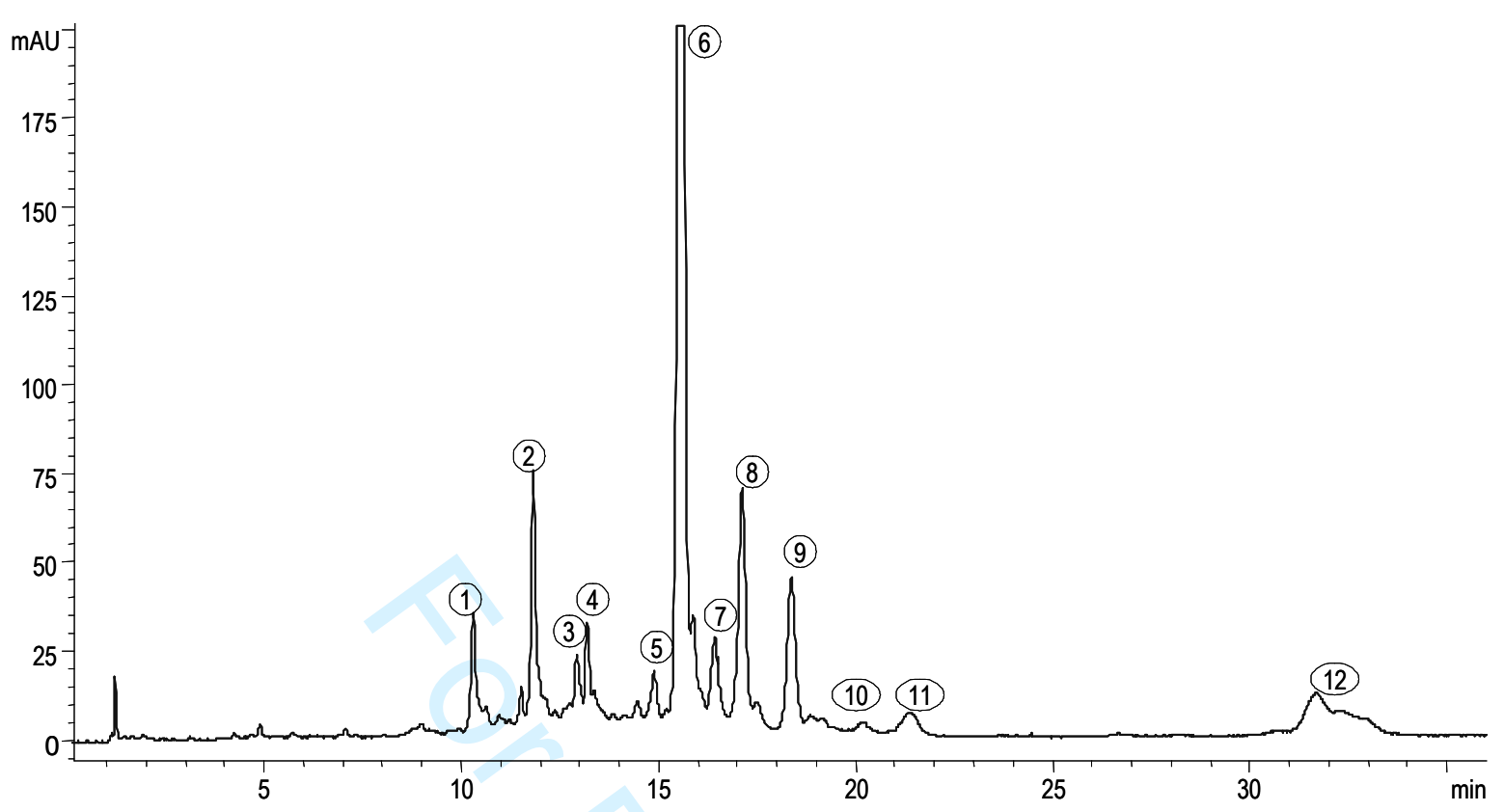

Figure 4. 

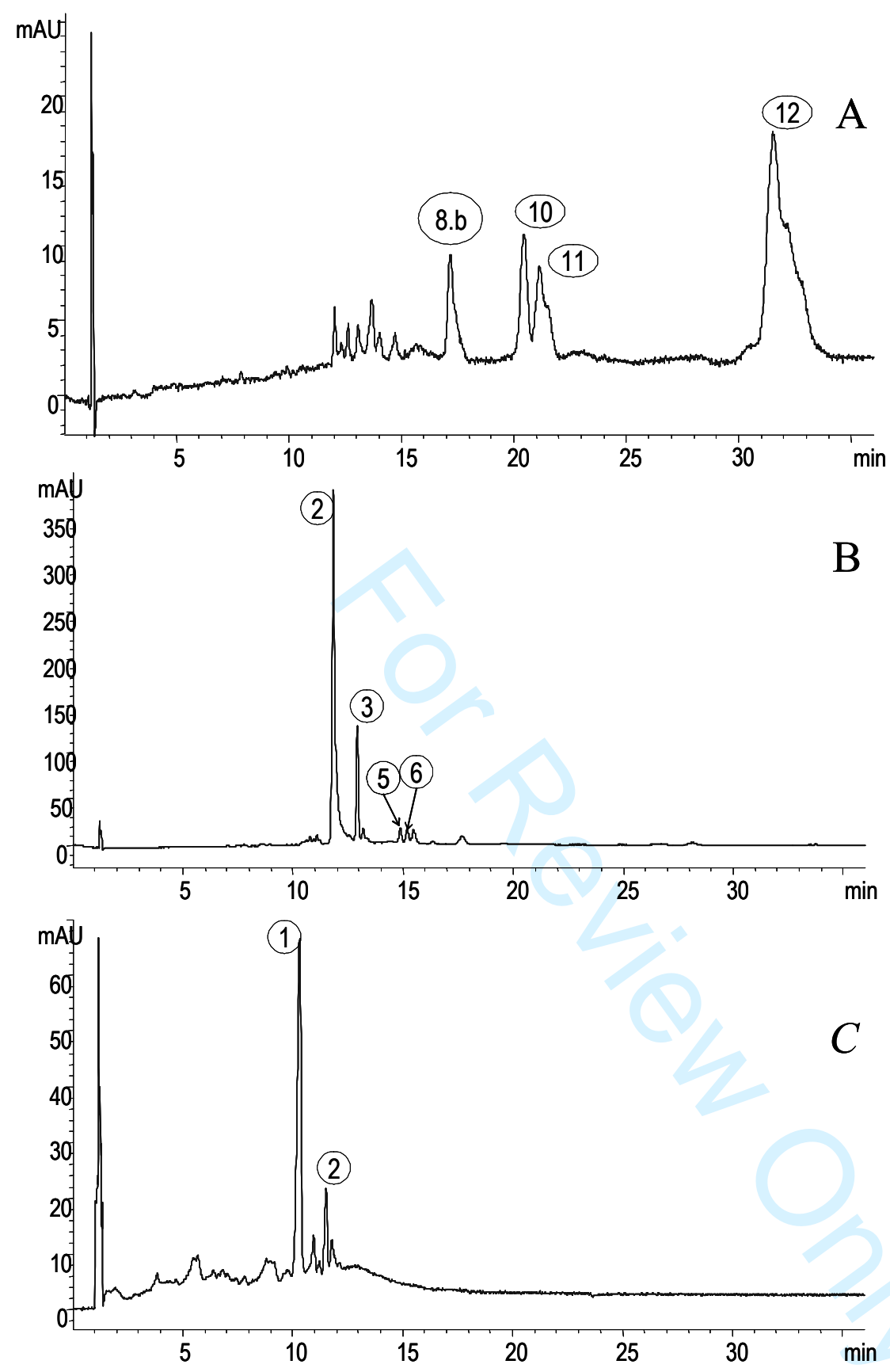

Figure 5. 

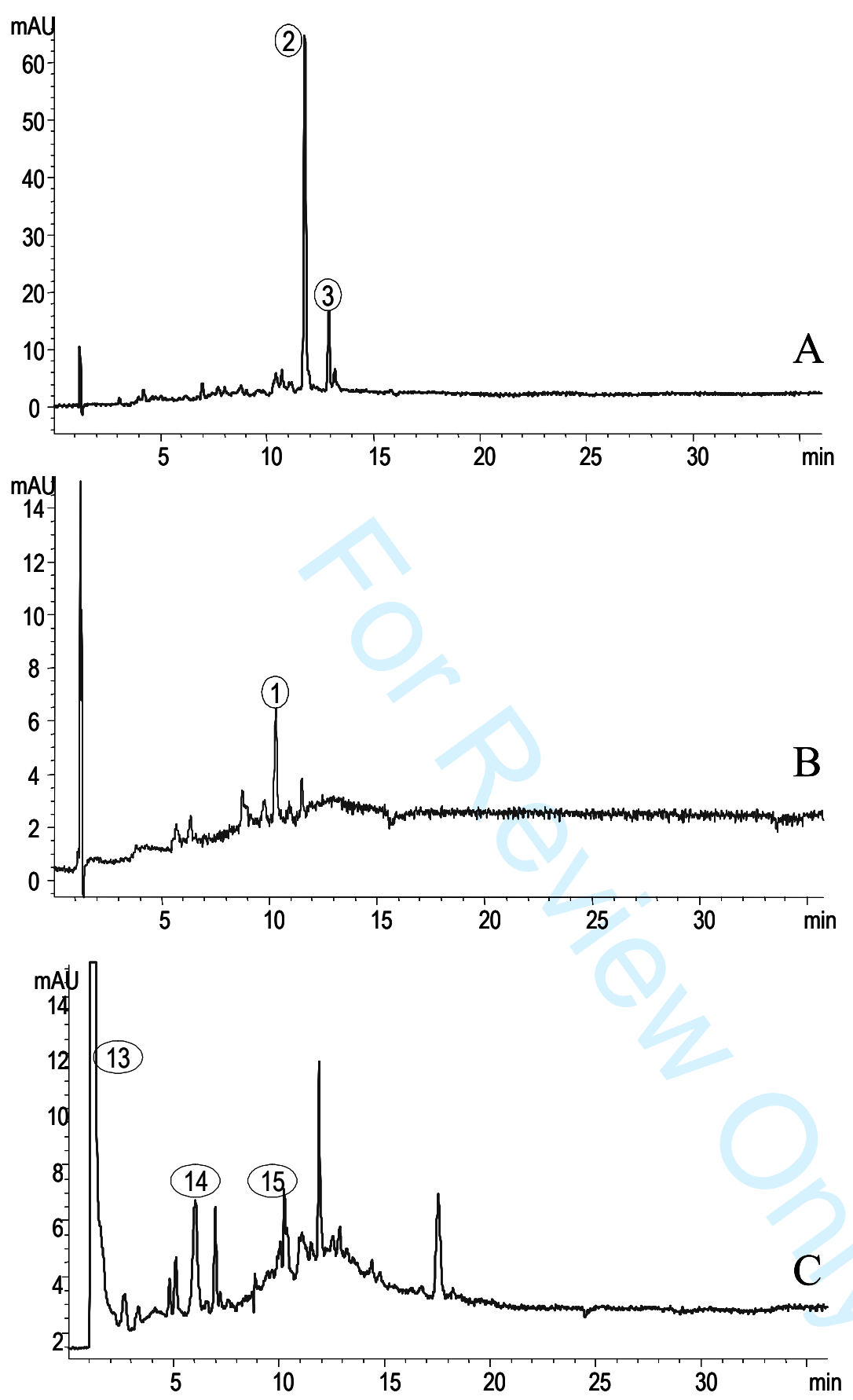

Figure 6. 

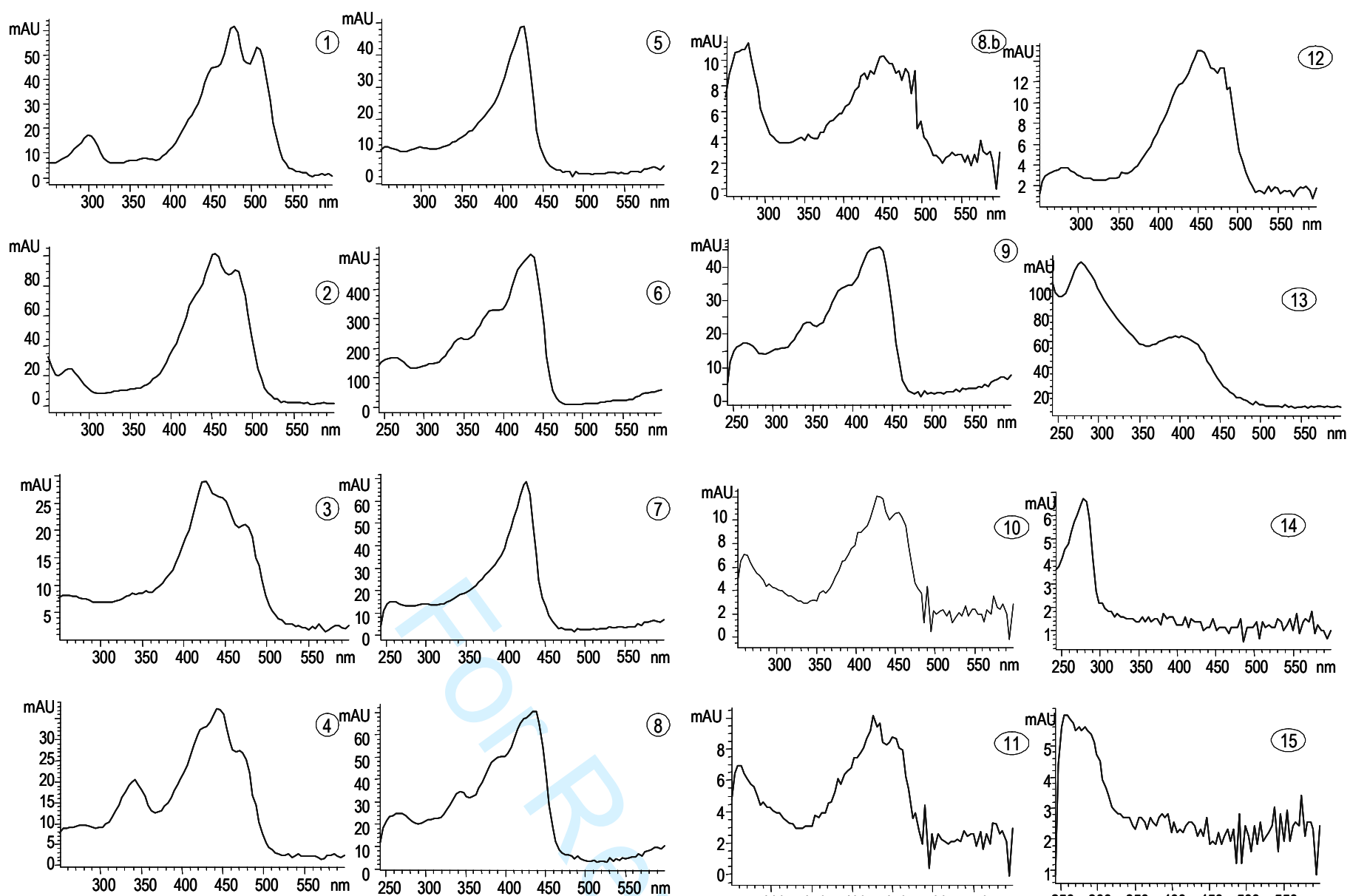

8.
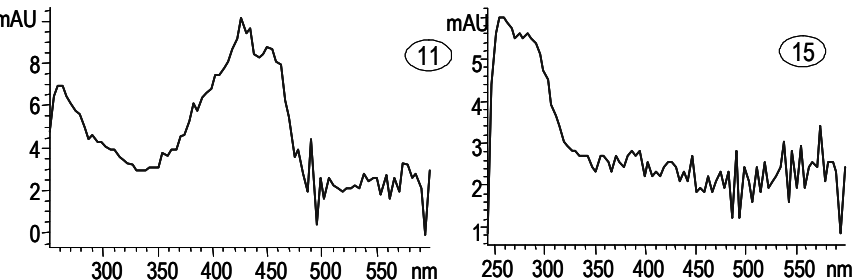

Figure 7. 